\title{
Shapiro's lemma for topological K-theory of groups
}

\author{
Jérôme Chabert, Siegfried Echterhoff and Hervé Oyono-Oyono
}

\begin{abstract}
Let $X \rtimes G$ be the crossed product groupoid of a locally compact group $G$ acting on a locally compact space $X$. For any $X \rtimes G$-algebra $A$ we show that a natural forgetful map from the topological K-theory $\mathrm{K}_{*}^{\text {top }}(X \rtimes G ; A)$ of the groupoid $X \rtimes G$ with coefficients in $A$ to the topological K-theory $\mathrm{K}_{*}^{\text {top }}(G ; A)$ of $G$ with coefficients in $A$ is an isomorphism. We then discuss several interesting consequences of this result for the Baum-Connes conjecture.
\end{abstract}

Mathematics Subject Classification (2000). 19K35, 19KXX, 46L80 (Primary), 22D15, 22A22 (Secondary).

Keywords. Topological K-theory, crossed-product groupoid, Baum-Connes conjecture.

\section{Introduction and statement of the main results}

Let $G$ be a locally compact group. By a $G$-algebra we understand a $C^{*}$-algebra $A$ equipped with a (strongly) continuous action of $G$ on $A$. We can follow [3] (but see also the discussions in [6]) to compute the topological K-theory of the group $G$ with coefficients in $A$ as

$$
\mathrm{K}_{*}^{\mathrm{top}}(G ; A)=\lim _{Z \subseteq \mathcal{E}(G)} \mathrm{KK}_{*}^{G}\left(C_{0}(Z), A\right),
$$

where $\mathcal{E}(G)$ denotes a universal example for the proper actions of $G, Z$ runs through the $G$-compact subsets of $\mathcal{E}(G)$ (i.e., $Z$ is a $G$-invariant subset of $\mathcal{E}(G)$ such that $G \backslash Z$ is compact), and $\mathrm{KK}_{*}^{G}\left(C_{0}(Z), A\right)$ denotes Kasparov's equivariant KK-theory (see [10]). The topological K-theory forms the left hand side of the Baum-Connes assembly map

$$
\mu_{G, A}: \mathrm{K}_{*}^{\mathrm{top}}(G ; A) \rightarrow \mathrm{K}_{*}\left(A \rtimes_{r} G\right),
$$

where $A \rtimes_{r} G$ denotes the reduced crossed product of $A$ and $G$. The BaumConnes conjecture asserts that $\mu_{G, A}$ should always be an isomorphism. If this is true for a given pair $(G, A)$, we say that $G$ satisfies $B C$ for $A$. Note that it seems now clear that the conjecture does not hold for all pairs $(G, A)$ (e.g., see

This work was partially supported by the Deutsche Forschungsgemeinschaft (SFB 478). 
[9]), but the conjecture has been shown to be valid for a fairly large class of groups and coefficient algebras (for general surveys on recent results we refer to [17, 24]). Perhaps, the strongest positive result has been obtained by Higson and Kasparov in [8], where they show that every group with the Haagerup property, and, in particular, all amenable groups satisfy BC for arbitrary coefficient algebras $A$.

The constructions of the topological K-theory for a group and the assembly map have been extended to locally compact groupoids $\mathcal{G}$ (with Haar system) and $\mathcal{G}$-algebras $A$ by Tu in [20], using Le Gall's notion of equivariant KK-theory for groupoids ([14]). Tu was also able to prove a groupoid version of the above mentioned result of Higson and Kasparov (see [21]). In particular, all topologically amenable groupoids (in the sense of [1]) satisfy BC for arbitrary coefficients.

In this paper we want to show that considering the conjecture for groupoids does indeed give extra information for groups. If $G$ is a locally compact group which acts continuously on a locally compact space $X$, then we can consider the crossed-product groupoid $X \rtimes G$ of $X$ by $G$. An $X \rtimes G$-algebra is a $G$-algebra $A$ together with a $G$-equivariant nondegenerate $*$-homomorphism $\phi: C_{0}(X) \rightarrow$ $Z M(A)$, where $Z M(A)$ denotes the center of the multiplier algebra of $A$. Since every $X \rtimes G$-algebra is also a $G$-algebra, we can consider both topological Ktheories

$$
\mathrm{K}_{*}^{\mathrm{top}}(G ; A) \quad \text { and } \quad \mathrm{K}_{*}^{\mathrm{top}}(X \rtimes G ; A) .
$$

Moreover, it follows easily from the definitions that the reduced crossed product $A \rtimes_{r} G$ coincides with the reduced groupoid crossed product $A \rtimes_{r}(X \rtimes G)$ for every $X \rtimes G$-algebra $A$. Thus, the Baum-Connes conjectures for $G$ and $X \rtimes G$ predict that both topological K-theories should coincide. Indeed, the main result of this paper is

Theorem 0.1. Let $A$ be an $X \rtimes G$-algebra. There exists a natural forgetful map

$$
\mathcal{F}: \mathrm{K}_{*}^{\mathrm{top}}(X \rtimes G ; A) \rightarrow \mathrm{K}_{*}^{\mathrm{top}}(G ; A),
$$

which is an isomorphism of groups.

We remark at this point that the result is fairly easy in case $X$ is compact (this case was used without proof in [7], but a more general groupoid version is given in [18, Lemma 4.1]).

We refer to $\S 1$ for more details on the construction of $\mathcal{F}$. The main consequence of Theorem 0.1 is to provide a way to connect the Baum-Connes conjecture for groups to the conjecture for groupoids:

Corollary 0.2. Let $X$ be a $G$-space and let $A$ be an $X \rtimes G$-algebra. Then $G$ satisfies $B C$ for $A$ if and only if $X \rtimes G$ satisfies $B C$ for $A$.

Recall that a $G$-algebra $A$ is called proper if $A$ is an $X \rtimes G$-algebra for some proper $G$-space $X$. Using the descent isomorphism [12, Theorem 5.4], we are now 
able to derive from the above corollary an elementary proof of [6, Théorème 2.2], which is a key technical result for the $\gamma$-element approach of the Baum-Connes conjecture:

Corollary 0.3. Assume that $A$ is a proper $G$-algebra. Then $G$ satisfies $B C$ for $A$.

Most important, this result can be generalized substantially: proper $G$-spaces are amenable, and as a direct application of Tu's groupoid version of the very deep Higson-Kasparov theorem (already mentioned above), we obtain

Corollary 0.4. Assume that $X$ is a topologically amenable $G$-space (in the sense of [1]) and let $A$ be an $X \rtimes G$-algebra. Then the Baum-Connes assembly map

$$
\mu_{G, A}: \mathrm{K}_{*}^{\mathrm{top}}(G ; A) \rightarrow \mathrm{K}_{*}\left(A \rtimes_{r} G\right)
$$

is an isomorphism.

Another important application uses equivalences of groupoids: Assume that $X$ is a locally compact space equipped with commuting free and proper actions of the locally compact groups $H$ and $K$. Assume that $A$ is an $X \rtimes(H \times K)$-algebra and let $A^{K}$ (resp. $A^{H}$ ) denote the generalized fixed-point algebras of $A$ by $K$ (resp. $H)$ in the sense of Kasparov (see [10] and $\S 4$ below). Then $A^{K}$ is a $(K \backslash X) \rtimes H$ algebra and $A^{H}$ is an $(H \backslash X) \rtimes K$-algebra. It follows from results of Kasparov [10] (which have been generalized to general groupoid equivalences by Le Gall in [14]) that there are natural isomorphisms between the topological K-theory groups

$\mathrm{K}_{*}^{\text {top }}\left((H \backslash X) \rtimes K ; A^{H}\right), \quad \mathrm{K}_{*}^{\text {top }}(X \rtimes(H \times K) ; A) \quad$ and $\quad \mathrm{K}_{*}^{\text {top }}\left((K \backslash X) \rtimes H ; A^{K}\right)$.

Applying the forgetful map to all theses groups we obtain similar isomorphisms between

$$
\mathrm{K}_{*}^{\mathrm{top}}\left(K ; A^{H}\right), \quad \mathrm{K}_{*}^{\mathrm{top}}(H \times K ; A) \quad \text { and } \quad \mathrm{K}_{*}^{\mathrm{top}}\left(H ; A^{K}\right) .
$$

Since the crossed products by the above actions are all Morita equivalent by $[10,15]$, they also have the same K-theories. Thus, by checking that the various assembly maps are compatible with these isomorphisms we obtain

Theorem 0.5. Let $X, H, K$ and $A$ be as above. Then the following are equivalent:

(i) $H \times K$ satisfies $B C$ for $A$.

(ii) $H$ satisfies $B C$ for $A^{K}$.

(iii) $K$ satisfies $B C$ for $A^{H}$.

As a special case of this, we obtain a new and conceptionally easier proof of one of the main results of [5]: Assume that $H$ is a closed subgroup of the locally compact group $G$ and that $B$ is an $H$-algebra. Then the induced algebra $\operatorname{Ind}_{H}^{G} B$ can be defined as the generalized fixed-point algebra $\left(C_{0}(G) \otimes B\right)^{H}$ with respect to the diagonal $H$-action (using right translation of $H$ on $G$ ). Thus, putting 
$A=C_{0}(G) \otimes B$ and $K=G$ (acting by left translation on $C_{0}(G)$ ), the above results provide

Corollary 0.6 ( $\operatorname{cf}[5$, Theorem 2.5]). Let $H, G$ and $B$ be as above. Then $H$ satisfies $B C$ for $B$ if and only if $G$ satisfies $B C$ for $\operatorname{Ind}_{H}^{G} B$. In particular, if $G$ satisfies $B C$ for all coefficient algebras, the same is true for $H$.

Note that the main ideas for the proof of Theorem 0.1 follow from the general methods as developed in [5]: we use $\mathcal{E}(G) \times \mathcal{E}\left(G / G_{0}\right)$, equipped with the diagonal action of $G$, as a universal example for the proper actions of $G\left(G_{0}\right.$ denotes the component of the identity of $G)$. Since $G / G_{0}$ is totally disconnected, $\mathcal{E}\left(G / G_{0}\right)$ can be realized as a locally finite simplicial complex such that the $G$-stabilizers of the single simplices are almost connected. This allows, using compression and a Mayer-Vietoris argument, to reduce the problem to the case of almost connected groups (i.e., $G / G_{0}$ is compact). Since such groups possess a $\gamma$-element by [10], a fairly simple argument shows the validity of Theorem 0.1 in this case. Note that all locally compact groups and spaces appearing in this paper are assumed to be second countable and Hausdorff, and all coefficient algebras are assumed to be separable. Moreover, all tensor products of $C^{*}$-algebras are assumed to be spatial. The authors are very grateful to Jean Renault and George Skandalis for some useful conversations on the topic.

Most of this work was done while the second author visited the Mathematics Department at Université Blaise Pascal in Clermont-Ferrand. The second author is very grateful to the members of the department (in particular to Jerome Chabert and Hervé Oyono-Oyono) for their warm hospitality.

\section{Some preliminaries and construction of the forgetful map}

Let $G$ be a locally compact group acting continuously on a locally compact space $X$. The crossed-product groupoid $X \rtimes G$ consists of all pairs $(x, g)$ with $x \in X, g \in$ $G$. Its base space is $X(\cong X \times\{e\})$, and the source and range maps are given by

$$
s((x, g))=g^{-1} x, \quad r((x, g))=x .
$$

The composition law is $(g x, g)\left(x, g^{\prime}\right)=\left(g x, g g^{\prime}\right)$ and the inversion is $(x, g)^{-1}=$ $\left(g^{-1} x, g^{-1}\right)$.

An action of the groupoid $X \rtimes G$ on a topological space $Z$ is given by a continuous action of $G$ on $Z$ together with a continuous $G$-equivariant map $p: Z \rightarrow X$. Such an action is proper if and only if the underlying group action of $G$ on $Z$ is proper, i.e., if and only if the structural map $G \times Z \rightarrow Z \times Z ;(g, z) \mapsto(g \cdot z, z)$ is proper in the sense that inverse images of compact sets are compact. A map $\phi: Y \rightarrow Z$ between two $X \rtimes G$-spaces $Y$ and $Z$ is $X \rtimes G$-equivariant if $\phi$ is $G$-equivariant and $p_{Z} \circ \phi=p_{Y}$, where $p_{Y}: Y \rightarrow X$ and $p_{Z}: Z \rightarrow X$ denote the underlying base maps. 
Recall that a universal example, $\mathcal{E}(G)$, for the proper actions of $G$ is a locally compact proper $G$-space such that for any other locally compact proper $G$-space $Z$ there exists a continuous $G$-equivariant map $F: Z \rightarrow \mathcal{E}(G)$ which is unique up to $G$-homotopy (see $[12,6]$ ). Note that $\mathcal{E}(G)$ always exists $([12])$, and it follows from the definition that it is unique up to $G$-homotopy. It is straightforward to extend this notion of universal proper space to the setting of groupoid actions (see [20]), but here we only need it for the crossed-product groupoid $X \rtimes G$. For this we let $X \rtimes G$ act on $\mathcal{E}(G) \times X$ via the diagonal action of $G$ and the (G-equivariant) second projection $\pi: \mathcal{E}(G) \times X \rightarrow X$. This clearly defines a proper action of $X \rtimes G$ on $\mathcal{E}(G) \times X$ and we get:

Lemma 1.1. Equipped with the action defined above, $\mathcal{E}(G) \times X$ is a universal example for the proper actions of the groupoid $X \rtimes G$.

Proof. Let $Z$ be a proper $X \rtimes G$ space with base map $p: Z \rightarrow X$. Then the group $G$ acts properly on $Z$ and since $\mathcal{E}(G)$ is a universal example for proper actions of $G$, there exists a continuous equivariant map $\varphi: Z \rightarrow \mathcal{E}(G)$. The map $Z \ni z \mapsto \phi(z)=(\varphi(z), p(z)) \in \mathcal{E}(G) \times X$ is then $X \rtimes G$-equivariant. Now if $z \mapsto \phi^{\prime}(z)=\left(q_{1}(z), q_{2}(z)\right)$ is another $X \rtimes G$-equivariant map, then clearly $q_{2}=p$. The universal property of $\mathcal{E}(G)$ with respect to proper $G$-spaces implies that $q_{1}$ and $\varphi$ are $G$-equivariantly homotopic. Both facts imply that $\phi$ and $\phi^{\prime}$ are $X \rtimes G$ homotopic.

Recall from [20] that the topological K-theory of a locally compact groupoid $\mathcal{G}$ with coefficients in the $\mathcal{G}$-algebra $A$ is defined as

$$
\mathrm{K}_{*}^{\mathrm{top}}(\mathcal{G} ; A)=\lim _{Y \subseteq \mathcal{E}(\mathcal{G})} \mathrm{KK}_{*}^{\mathcal{G}}\left(C_{0}(Y), A\right),
$$

where $Y$ runs through the $\mathcal{G}$-compact subsets of $\mathcal{E}(\mathcal{G})$ and $\mathrm{KK}_{*}^{\mathcal{G}}$ denotes Le Gall's equivariant KK-theory (see [14]). In case of the crossed-product groupoid $X \rtimes G$,

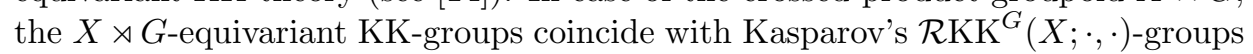
(see $[14, \S 5]$ and $[10, \S 3])$. Since a subset of $\mathcal{E}(G) \times X$ is $X \rtimes G$-compact if and only if it is $G$-compact we obtain

$$
\mathrm{K}_{*}^{\mathrm{top}}(X \rtimes G ; A)=\lim _{Y \subseteq \mathcal{E}(G) \times X} \mathcal{R} \mathrm{KK}_{*}^{G}\left(X ; C_{0}(Y), A\right),
$$

for any $X \rtimes G$-algebra $A$, where $Y$ runs through the $G$-compact subsets of $\mathcal{E}(G) \times X$. Recall that for any pair of $X \rtimes G$-algebras $(B, A)$, the cycles for $\mathcal{R} K^{G}(X ; B, A)$ consist of equivariant Kasparov triples $(\mathcal{E}, \Phi, T)$, as in the construction of $\mathrm{KK}^{G}(B, A)$, but which satisfy the additional requirement that

$$
(h \cdot a) \cdot(e \cdot b)=(a \cdot e) \cdot(b \cdot h) \quad \text { for all } h \in C_{0}(X), a \in A, e \in \mathcal{E} \text { and } b \in B .
$$

Thus, forgetting this extra requirement gives a natural homomorphism

$$
f: \mathcal{R K K}_{*}^{G}(X ; B, A) \rightarrow \mathrm{KK}_{*}^{G}(B, A) .
$$


In particular, for any $G$-compact subset $Y \subseteq \mathcal{E}(G) \times X$ we obtain a canonical composition of maps

$$
\mathcal{R K K}_{*}^{G}\left(X ; C_{0}(Y), A\right) \stackrel{f}{\rightarrow} \mathrm{KK}_{*}^{G}\left(C_{0}(Y), A\right) \stackrel{\pi_{1 *}}{\rightarrow} \mathrm{KK}_{*}^{G}\left(C_{0}\left(\pi_{1}(Y)\right), A\right) \rightarrow \mathrm{K}_{*}^{\mathrm{top}}(G ; A),
$$

where $\pi_{1}: \mathcal{E}(G) \times X \rightarrow \mathcal{E}(G)$ denotes the canonical projection, which restricts to a proper map on the $G$-compact set $Y$, and the last map follows from the definition of the topological K-theory. Let us denote the above composition by

$$
\mathcal{F}_{Y}: \mathcal{R K K}_{*}^{G}\left(X ; C_{0}(Y), A\right) \rightarrow \mathrm{K}_{*}^{\mathrm{top}}(G ; A) .
$$

It is straightforward to check that the maps $\mathcal{F}_{Y}$ are compatible with taking limits, and hence they induce a well defined group homomorphism

$$
\mathcal{F}: \mathrm{K}_{*}^{\mathrm{top}}(X \rtimes G ; A) \rightarrow \mathrm{K}_{*}^{\mathrm{top}}(G ; A),
$$

which we call the forgetful map. The statement of our main theorem (Theorem 0.1 ) says that $\mathcal{F}$ is always an isomorphism.

If $A$ is an $X \rtimes G$-algebra, then it follows from the definitions that the reduced groupoid crossed product $A \rtimes_{r}(X \rtimes G)$ coincides with the reduced crossed product $A \rtimes_{r} G$ of $A$ by $G$ (for a concise discussion of the definition of crossed products by groupoids we refer to $[18, \S 2.4])$. Thus we get two assembly maps

$\mu_{G, A}: \mathrm{K}_{*}^{\mathrm{top}}(G ; A) \rightarrow \mathrm{K}_{*}\left(A \rtimes_{r} G\right) \quad$ and $\quad \mu_{X \rtimes G, A}: \mathrm{K}_{*}^{\mathrm{top}}(X \rtimes G ; A) \rightarrow \mathrm{K}_{*}\left(A \rtimes_{r} G\right)$.

We need:

Lemma $1.2(\operatorname{cf}[18$, Lemma 4.1]). The diagram

$$
\mathrm{K}_{*}^{\mathrm{top}}(X \rtimes G ; A) \stackrel{\mathcal{F}}{\longrightarrow} \mathrm{K}_{*}^{\mathrm{top}}(G ; A)
$$

commutes.

Proof. The assembly map for $X \rtimes G$ is defined inductively via the maps

$$
\mu_{Y}: \mathcal{R K K}_{*}^{G}\left(X ; C_{0}(Y), A\right) \rightarrow \mathrm{K}_{*}\left(A \rtimes_{r} G\right),
$$

which, for any $G$-compact subset $Y \subseteq \mathcal{E}(G) \times X$, can be defined as the composition

$$
\begin{aligned}
& \mathcal{R K K}_{*}^{G}\left(X ; C_{0}(Y), A\right) \stackrel{f}{\rightarrow} \mathrm{KK}_{*}^{G}\left(C_{0}(Y), A\right) \stackrel{J_{G}}{\rightarrow} \mathrm{KK}_{*}\left(C_{0}(Y) \rtimes_{r} G, A \rtimes_{r} G\right) \\
& \stackrel{\Lambda_{Y} \otimes}{\longrightarrow} \mathrm{K}_{*}\left(A \rtimes_{r} G\right),
\end{aligned}
$$

where $\Lambda_{Y} \in \mathrm{K}_{0}\left(C_{0}(Y) \rtimes_{r} G\right)$ denotes the fundamental K-theory class corresponding to the proper and $G$-compact $G$-space $Y$ (e.g., see [4] and [20] for more details). 
Similarly, the assembly map for $G$ is defined inductively on the $G$-compact subsets $Z$ of $\mathcal{E}(G)$ by the compositions

$$
\mathrm{KK}_{*}^{G}\left(C_{0}(Z), A\right) \stackrel{J_{G}}{\rightarrow} \mathrm{KK}_{*}\left(C_{0}(Z) \rtimes_{r} G, A \rtimes_{r} G\right) \stackrel{\Lambda_{Z} \otimes}{\longrightarrow} \mathrm{K}_{*}\left(A \rtimes_{r} G\right),
$$

where $\Lambda_{Z}$ denotes the fundamental class of $Z$ in $\mathrm{K}_{*}\left(C_{0}(Z) \rtimes_{r} G\right)$. By construction, this fundamental class is natural with respect to $G$-equivariant continuous mapping, so one has:

$$
\Lambda_{Y}=\Lambda_{\pi_{1}(Y)} \otimes J_{G}\left(\pi_{1 *}\right)
$$

This implies that the diagram

$$
\begin{array}{ccc}
\mathrm{KK}_{*}\left(C_{0}(Y) \rtimes G, A \rtimes_{r} G\right) \stackrel{J_{G}\left(\pi_{1 *}\right)}{\longrightarrow} & \mathrm{KK}_{*}\left(C_{0}\left(\pi_{1}(Y)\right) \rtimes G, A \rtimes_{r} G\right) \\
\Lambda_{Y} \otimes \cdot \downarrow & \downarrow \Lambda_{\pi_{1}(Y)} \otimes . \\
\mathrm{K}_{*}\left(A \rtimes_{r} G\right) & =\mathrm{K}_{*}\left(A \rtimes_{r} G\right)
\end{array}
$$

commutes, which is enough to get the commutativity of (1.2).

\section{The compression isomorphism in $\mathcal{R K K}^{G}(X, \cdot, \cdot)$}

For the proof of Theorem 0.1, we need an $\mathcal{R} K K$-version of the compression isomorphism as given in [5, Proposition 5.14]. Recall that if $C$ is a closed subgroup of $G$ and $A$ is a $C$-algebra, then the induced $C^{*}$-algebra $\operatorname{Ind}_{C}^{G} A$ is defined as the space

$$
\begin{aligned}
& \left\{F \in C_{b}(G, A): F(s c)=c^{-1}(F(s)) \text { for all } s \in G, c \in C\right. \\
& \text { and } \left.(s C \mapsto\|F(s)\|) \in C_{0}(G / C)\right\},
\end{aligned}
$$

equipped with the pointwise operations and the supremum norm. The induced action of $G$ on $\operatorname{Ind}_{C}^{G} A$ is given by $s \cdot F(t)=F\left(s^{-1} t\right)$.

Remark 2.1. Assume that $C$ is a closed subgroup of $G, X$ is a locally compact $G$ space and $A$ is a $C$-algebra such that $\operatorname{Ind}_{C}^{G} A$ has the structure of an $X \rtimes G$-algebra. Then $A$ has a canonical structure as an $X \rtimes C$-algebra, where the $C_{0}(X)$-action on $A$ is given by the composition

$$
C_{0}(X) \stackrel{\phi}{\longrightarrow} Z M\left(\operatorname{Ind}_{C}^{G} A\right) \stackrel{\epsilon_{e}}{\longrightarrow} Z M(A) .
$$

Here $\phi: C_{0}(X) \rightarrow Z M\left(\operatorname{Ind}_{C}^{G} A\right)$ is the given structure map for $\operatorname{Ind}_{C}^{G}(A)$, and $\epsilon_{e}: Z M\left(\operatorname{Ind}_{C}^{G} A\right) \rightarrow Z M(A)$ denotes the morphism induced by the evaluation Ind $_{C}^{G} A \rightarrow A ; F \mapsto F(e)$ at the unit element $e$ of $G$. Since $\epsilon_{e}$ is $C$-equivariant, it follows that the above composition is also $C$-equivariant. Moreover, for $f \in C_{0}(X)$, 
$F \in \operatorname{Ind}_{C}^{G} A$, and $s \in G$, we get the equation:

$$
\begin{aligned}
(f \cdot F)(s) & =\left(s^{-1}(f \cdot F)\right)(e)=\left(s^{-1}(f) \cdot s^{-1}(F)\right)(e)=s^{-1}(f) \cdot\left(s^{-1}(F)(e)\right) \\
& =s^{-1}(f) \cdot(F(s)) .
\end{aligned}
$$

Conversely, if $X$ is a $G$-space and $A$ is an $X \rtimes C$-algebra with $C$-equivariant structure map $\psi: C_{0}(X) \rightarrow Z M(A)$, then the induced algebra $\operatorname{Ind}_{C}^{G} A$ becomes an $X \rtimes G$-algebra with structure map

$$
\operatorname{Ind} \psi: C_{0}(X) \rightarrow Z M\left(\operatorname{Ind}_{C}^{G} A\right) ; \quad(\operatorname{Ind} \psi(f) F)(s)=\psi\left(s^{-1}(f)\right)(F(s)),
$$

and it is clear that, via evaluation at $e$ in $G$, this induces the given $C_{0}(X)$-structure on $A$. It follows then from (2.1) that the above procedures give us a one-to-one correspondence between the $G$-equivariant $C_{0}(X)$-structures on $\operatorname{Ind}_{C}^{G} A$ and the $C$-equivariant $C_{0}(X)$-structures on $A$.

Assume now that $C$ is open in $G$. Using (2.1) it is easy to check that we get an $X \rtimes C$-equivariant inclusion $\iota_{A}: A \rightarrow \operatorname{Ind}_{C}^{G} A$ defined by

$$
\iota_{A}(a)(s)=\left\{\begin{array}{cc}
s^{-1}(a) & \text { if } s \in C \\
0 & \text { if } s \notin C
\end{array}\right\} .
$$

Thus, for any $X \rtimes G$-algebra $B$ we get a natural composition of maps in $\mathcal{R}$ KKtheory

$$
\mathcal{R K K}_{*}^{G}\left(X ; \operatorname{Ind}_{C}^{G} A, B\right) \stackrel{\operatorname{res}_{C}^{G}}{\longrightarrow} \mathcal{R} \operatorname{KK}_{*}^{C}\left(X ; \operatorname{Ind}_{C}^{G} A, B\right) \stackrel{\iota_{A}^{*}}{\longrightarrow} \mathcal{R} \mathrm{KK}_{*}^{C}(X ; A, B),
$$

which we shall call the compression map denoted by

$$
\operatorname{comp}_{C}^{G}: \mathcal{R K K}_{*}^{G}\left(X ; \operatorname{Ind}_{C}^{G} A, B\right) \rightarrow \mathcal{R K K}_{*}^{C}(X ; A, B) .
$$

Proposition 2.2 (cf. [5, Proposition 5.14]). Assume that $C$ is an open subgroup of $G$. Let $X$ be a locally compact $G$-space and let $A$ be an $X \rtimes C$-algebra. Then the compression map $\operatorname{comp}_{C}^{G}: \mathcal{R K K}_{*}^{G}\left(X ; \operatorname{Ind}_{C}^{G} A, B\right) \rightarrow \mathcal{R} \mathrm{KK}_{*}^{C}(X ; A, B)$ is an isomorphism.

Proof. We only have to check that the constructions given in the proof of $[5$, Proposition 5.14] are compatible with the given $C_{0}(X)$-structures. First of all, we can replace $A$ by $A \otimes \mathcal{K}\left(L^{2}(C)\right)$ using the $X \rtimes C$-equivariant Morita equivalence $\left(A \otimes L^{2}(C), \alpha \otimes \lambda\right)$ between $A$ and $A \otimes \mathcal{K}\left(L^{2}(C)\right)$, where $\lambda$ denotes the left regular representation of $C$. The second diagram in the proof of [5, Proposition 5.14] then clearly commutes in $\mathcal{R}$ KK-theory. Thus, using the $\mathcal{R}$ KK-version of [13, Proposition 3.2] (which is allowed by the remarks in [13, §9]), we can assume without loss of generality that all elements (and homotopies) in $\mathcal{R} \mathrm{KK}^{C}(X ; A, B)$ are represented by Kasparov triples $(\mathcal{E}, \Phi, T)$ such that $\Phi(A) \mathcal{E}=\mathcal{E}$ and $T$ is a $C$-equivariant operator on $\mathcal{E}$. Having this, we can define a map

$$
\inf _{C}^{G}: \mathcal{R K K}^{C}(X ; A, B) \rightarrow \mathcal{R} \mathrm{KK}^{G}\left(X ; \operatorname{Ind}_{C}^{G} A, B\right)
$$


by defining $\inf _{C}^{G}([(\mathcal{E}, \Phi, T)])=[(\tilde{\mathcal{E}}, \tilde{\Phi}, \tilde{T})]$, with $(\tilde{\mathcal{E}}, \tilde{\Phi}, \tilde{T})$ given by exactly the same formulas as used in [5, Proposition 5.14]. Using the computations of [5], it follows that $\inf _{C}^{G}$ is well defined if we can show that the module $\tilde{\mathcal{E}}$ preserves the $C_{0}(X)$-structures, that is

$$
f \cdot \xi=\xi \cdot f
$$

for all $f \in C_{0}(X), \xi \in \tilde{\mathcal{E}}$, where the left and right actions of $C_{0}(X)$ an $\tilde{\mathcal{E}}$ are induced from the left and right actions of $\operatorname{Ind}_{C}^{G} A$ and $B$. For this, recall that we have a dense subspace in $\tilde{\mathcal{E}}$ consisting of continuous functions $\xi: G \rightarrow \mathcal{E}$ such that

- $\xi(s c)=c^{-1}(\xi(s))$ for all $s \in G$ and $c \in C$;

- the map $s C \mapsto\|\xi(s)\|$ has finite support in $G / C$.

Moreover, the left and right actions of $\operatorname{Ind}_{C}^{G} A$ and $B$ on $\tilde{\mathcal{E}}$ are given on such functions $\xi$ by the formulas

$(\tilde{\Phi}(F) \xi)(s)=\Phi(F(s))(\xi(s)) \quad$ and $\quad(\xi \cdot b)(s)=\xi(s) \cdot s^{-1}(b), \quad F \in \operatorname{Ind}_{C}^{G} A, b \in B$.

Thus, using Remark 2.1, we can compute for $f \in C_{0}(X)$ :

$$
(f \cdot \xi)(s)=s^{-1}(f) \cdot(\xi(s))=(\xi(s)) \cdot s^{-1}(f)=(\xi \cdot f)(s) .
$$

By continuity, the equation $f \cdot \xi=\xi \cdot f$ then holds for all $\xi \in \tilde{\mathcal{E}}$. Exactly the same arguments as used in [5, Proposition 5.14] then show that $\inf _{C}^{G}$ is an inverse for $\operatorname{comp}_{C}^{G}$.

\section{Proof of the forgetful isomorphism}

We start by proving Theorem 0.1 for groups having a $\gamma$-element in the sense of [10] and [20] (the precise requirements for a $\gamma$-element in the group case are spelled out in [4, Definition 6.3]; the requirements in the groupoid case are similar). It follows from [10, Theorem 5.7] that every almost connected group $G$ (i.e., $G / G_{0}$ is compact) has a $\gamma$-element. In what follows,

$$
\sigma_{D}: \mathrm{KK}_{*}^{G}(A, B) \rightarrow \mathrm{KK}_{*}^{D}(A \otimes D, B \otimes D)
$$

denotes the canonical homomorphism given by tensoring with the $C^{*}$-algebra $D$, and

$$
J_{G}: \mathrm{KK}_{*}^{G}(A, B) \rightarrow \mathrm{KK}_{*}\left(A \rtimes_{r} G, B \rtimes_{r} G\right)
$$

denotes Kasparov's descent homomorphism in $\mathrm{KK}^{G}$-theory.

Lemma 3.1. Assume that $G$ has a $\gamma$-element. Then the forgetful map $\mathcal{F}$ is an isomorphism between $\mathrm{K}_{*}^{\mathrm{top}}(X \rtimes G ; A)$ and $\mathrm{K}_{*}^{\mathrm{top}}(G ; A)$.

Proof. If $G$ has a $\gamma$-element $\gamma_{G} \in \mathrm{KK}_{0}^{G}(\mathbb{C}, \mathbb{C})$ in the sense of [10], then $\mu_{G, A}$ is injective and its range is the $\gamma$-part

$$
\gamma_{G} \cdot \mathrm{K}_{*}\left(A \rtimes_{r} G\right):=\left\{J_{G}\left(\sigma_{A}\left(\gamma_{G}\right)\right) \otimes x: x \in \mathrm{K}_{*}\left(A \rtimes_{r} G\right)\right\}
$$


of $\mathrm{K}_{*}\left(A \rtimes_{r} G\right)$ (see [20, Proposition 5.23]). It is easy to check that $\gamma_{X \rtimes G}=$ $\sigma_{C_{0}(X)}(\gamma)$, viewed as an element of $\mathcal{R} \mathrm{KK}_{0}^{G}\left(X ; C_{0}(X), C_{0}(X)\right)$, is a $\gamma$-element for $X \rtimes G$ in the sense of [20], so $\mu_{X \rtimes G, A}$ is also injective with range equal to the $\gamma$-part

$$
\gamma_{X \rtimes G} \cdot \mathrm{K}_{*}\left(A \rtimes_{r} G\right):=\left\{J_{G}\left(\sigma_{A}^{X}\left(\gamma_{X \rtimes G}\right)\right) \otimes x: x \in \mathrm{K}_{*}\left(A \rtimes_{r} G\right)\right\},
$$

where here $\sigma_{A}^{X}: \mathcal{R K K}(X ; B, C) \rightarrow \mathcal{R K K}\left(X ; B \otimes_{C_{0}(X)} A, C \otimes_{C_{0}(X)} A\right)$ is given by taking balanced tensor products over $C_{0}(X)$ (see [10, Lemma 2.19]). Using the canonical isomorphism $C_{0}(X) \otimes_{C_{0}(X)} A \simeq A$, we can identify $\sigma_{A}^{X}\left(\gamma_{X \rtimes G}\right)$ ) with $\sigma_{A}\left(\gamma_{G}\right)$ and the result follows from Lemma 1.2.

We now extend the result to arbitrary locally compact groups. To do this we use the basic method of [5] by observing that if $\mathcal{E}(G)$ is a universal example for the proper actions of $G$ and $\mathcal{E}\left(G / G_{0}\right)$ is a universal example for the proper actions of $G / G_{0}$, where $G_{0}$ denotes the connected component of $G$, then $\mathcal{E}(G) \times \mathcal{E}\left(G / G_{0}\right)$, equipped with the diagonal $G$-action, is also a universal example for the proper actions of $G$. Since $G / G_{0}$ is totally disconnected, $\mathcal{E}\left(G / G_{0}\right)$ may be realized as a locally finite simplicial complex, which then allows to use a Mayer-Vietoris argument to reduce to the case of almost connected groups.

Notations 3.2. In the following, we write $N$ for the identity component $G_{0}$ of $G$. As in the discussion above, we take for $\mathcal{E}(G / N)$ the geometric realization of a locally finite simplicial complex on which $G / N$ acts properly, simplicially and respecting the types (see the discussion following [4, Lemma 7.10]). In particular, the interior $\stackrel{\circ}{W}$ of a single simplex is invariant under the action of an element $g \in G$ if and only if $g$ fixes every point $y \in \stackrel{\circ}{W}$.

Let $W$ denote a finite union of simplices in $\mathcal{E}(G / N)$, and let $K$ denote a fixed compact subset of $X$. Let $T=G \cdot(W \times K) \subseteq \mathcal{E}(G / N) \times X, Y=G \cdot W \subseteq \mathcal{E}(G / N)$ and $Z=G \cdot K \subseteq X$.

We denote by $\operatorname{dim} W$ the maximal dimension of the simplices in $W$, and we let $\stackrel{o}{W}$ be the union of the interiors of the simplices of $W$ of $\operatorname{dimension} \operatorname{dim} W$. Set $|W|=W \backslash \stackrel{\circ}{W},|Y|=G \cdot|W|$ and $|T|=T \backslash G \cdot(\stackrel{\circ}{W} \times K)=G \cdot(|W| \times K)$.

The following lemma is fundamental for the proof of Theorem 0.1 :

Lemma 3.3. The $C_{0}(X)$-linear epimorphism $C_{0}(T) \rightarrow C_{0}(|T|)$, given by restriction of functions, admits a $C_{0}(X)$-linear cross section $\sigma_{T}: C_{0}(|T|) \rightarrow C_{0}(T)$, which can be chosen norm decreasing, $G$-equivariant, and completely positive.

Proof. Such section can be constructed by using the linear contraction of the simplices of $W$ which are of dimension $\operatorname{dim} W$ onto their barycenters. If $W$ is a single simplex with vertices $v_{0}, v_{1}, \ldots v_{n}$ and barycenter $y_{0}=\sum_{i=0}^{n} \frac{1}{n+1} v_{i}$, we consider the $\operatorname{map} W \backslash\left\{y_{0}\right\} \rightarrow|W|$ which sends $y=\sum_{i=0}^{n} t_{i} v_{i}$ to $|y|=\frac{1}{1-(n+1) t} \sum_{i=0}^{n}\left(t_{i}-t\right) v_{i}$, 
where $t:=\min \left\{t_{0}, \ldots t_{n}\right\}$. In fact, $|y|$ is the projection of $y$ onto the nearest face of $W$. We can easily extend the definition of this map to get a $G$-equivariant map $G \cdot W \backslash G \cdot\left\{y_{0}\right\} \rightarrow G \cdot|W|$. If $W$ is a finite union of simplices, we may apply this procedure independently to each $G \cdot W_{i}$, where $W_{1}, \ldots, W_{k}$ denote the simplices of dimension $\operatorname{dim} W$ in $W$, to get a $G$-equivariant map $Y \backslash Y_{0} \rightarrow|Y|$, where $Y_{0}$ denotes the set of barycenters of the simplices $g \cdot W_{i}, g \in G, 1 \leq i \leq k$.

We now define $\sigma_{T}: C_{0}(|T|) \rightarrow C_{0}(T)$ by

$$
\begin{aligned}
& \sigma_{T}(f)(y, z) \\
& = \begin{cases}f(y, z) & \text { if } y \text { does not belong to a simplex of } \operatorname{dimension} \operatorname{dim} W ; \\
\frac{d\left(y, Y_{0}\right)}{d\left(|y|, Y_{0}\right)} \cdot f(|y|, z) & \text { if } y \text { belongs to a simplex of } \operatorname{dimension} \operatorname{dim} W \text { and } y \notin Y_{0} ; \\
0 & \text { if } y \in Y_{0},\end{cases}
\end{aligned}
$$

where $d\left(y, Y_{0}\right)$ denotes the euclidean distance between $y$ and the barycenter of the simplex of dimension $\operatorname{dim} W$ to which $y$ belongs.

By construction, we get that $\sigma_{T}$ is a norm decreasing completely positive section of the epimorphism $C_{0}(T) \rightarrow C_{0}(|T|)$, given by restriction of functions. It is also straitforward to check the $G$-equivariance and the $C_{0}(X)$-linearity of $\sigma_{T}$.

Using $\mathcal{E}(G) \times \mathcal{E}(G / N)$ as a realization of the universal proper $G$-space, we can apply Lemma 1.1 to obtain a realization of the universal proper $X \rtimes G$-space as $\mathcal{E}(G) \times \mathcal{E}(G / N) \times X$, all spaces being equipped with the diagonal $G$-action. This allows us to compute the topological K-theories of $G$ and $X \rtimes G$ with coefficients in the $X \rtimes G$-algebra $A$ by the formulas

$$
\begin{aligned}
& \mathrm{K}_{*}^{\mathrm{top}}(G ; A)=\lim _{L, W} \mathrm{KK}_{*}^{G}\left(C_{0}(G \cdot(L \times W)), A\right) \text { and } \\
& \mathrm{K}_{*}^{\mathrm{top}}(X \rtimes G ; A)=\lim _{L, W, K} \mathcal{R} \mathrm{KK}_{*}^{G}\left(X ; C_{0}(G \cdot(L \times W \times K)), A\right),
\end{aligned}
$$

where $L$ runs through the compact subsets of $\mathcal{E}(G), W$ runs through the finite subcomplexes of $\mathcal{E}(G / N)$, and $K$ runs through the compact subsets of $X$. We have to introduce some further notation:

Definition 3.4. For any given finite subcomplex $W \subseteq \mathcal{E}(G / N)$ we define

$$
\begin{aligned}
& \mathrm{K}_{*}^{\mathrm{top}}(G ; A)[W]:=\lim _{L} \mathrm{KK}_{*}^{G}\left(C_{0}(G \cdot(L \times W)), A\right) \quad \text { and } \\
& \mathrm{K}_{*}^{\mathrm{top}}(G ; A)[\stackrel{o}{W}]:=\lim _{L} \mathrm{KK}_{*}^{G}\left(C_{0}(G \cdot(L \times \stackrel{\circ}{W})), A\right),
\end{aligned}
$$

where $L$ runs through the compact subsets of $\mathcal{E}(G)$. Similarly, we define

$$
\begin{aligned}
& \mathrm{K}_{*}^{\mathrm{top}}(X \rtimes G ; A)[W]:=\lim _{L, K} \mathcal{R} \mathrm{KK}_{*}^{G}\left(X ; C_{0}(G \cdot(L \times W \times K)), A\right) \text { and } \\
& \mathrm{K}_{*}^{\mathrm{top}}(X \rtimes G ; A)[\stackrel{o}{W}]:=\lim _{L, K} \mathcal{R} \mathrm{KK}_{*}^{G}\left(X ; C_{0}(G \cdot(L \times \stackrel{o}{W} \times K)), A\right),
\end{aligned}
$$


where $L$ runs through the compact subsets of $\mathcal{E}(G)$ and $K$ runs through the compact subsets of $X$. Moreover, for any pair $(L, K)$ with $L \subseteq \mathcal{E}(G), K \subseteq X$ compact, we define the maps

$$
\begin{aligned}
& \mathcal{F}_{L, K}^{W}: \mathcal{R K K}_{*}^{G}\left(X ; C_{0}(G \cdot(L \times W \times K)), A\right) \rightarrow \mathrm{KK}_{*}^{G}\left(C_{0}(G \cdot(L \times W)), A\right) \text { and } \\
& \mathcal{F}_{L, K}^{W}: \mathcal{R K K}_{*}^{G}\left(X ; C_{0}(G \cdot(L \times \stackrel{o}{W} \times K)), A\right) \rightarrow \mathrm{KK}_{*}^{G}\left(C_{0}(G \cdot(L \times \stackrel{o}{W})), A\right)
\end{aligned}
$$

as in the definition of the forgetful map $\mathcal{F}$, i.e., we first "forget" the $C_{0}(X)$ action and then apply the KK-maps induced by the first projections $G \cdot(L \times W \times K) \rightarrow$ $G \cdot(L \times W)$ and $G \cdot(L \times \stackrel{o}{W} \times K) \rightarrow G \cdot(L \times \stackrel{o}{W})$, respectively (note that both maps are proper). The maps $\mathcal{F}_{L, K}^{W}$ and $\mathcal{F}_{L, K}^{W}$ are compatible with inductive limits over $L$ and $K$, so they induce well defined maps

$$
\begin{aligned}
& \mathcal{F}^{W}: \mathrm{K}_{*}^{\mathrm{top}}(X \rtimes G ; A)[W] \rightarrow \mathrm{K}_{*}^{\mathrm{top}}(G ; A)[W] \quad \text { and } \\
& \mathcal{F}^{\stackrel{o}{W}}: \mathrm{K}_{*}^{\mathrm{top}}(X \rtimes G ; A)[\stackrel{o}{W}] \rightarrow \mathrm{K}_{*}^{\mathrm{top}}(G ; A)[\stackrel{o}{W}] .
\end{aligned}
$$

It is clear that the topological K-theories of $G$ and $X \rtimes G$ with coefficients in $A$ can now be computed as

$\mathrm{K}_{*}^{\mathrm{top}}(G ; A)=\lim _{W} \mathrm{~K}_{*}^{\mathrm{top}}(G ; A)[W] \quad$ and $\quad \mathrm{K}_{*}^{\mathrm{top}}(X \rtimes G ; A)=\lim _{W} \mathrm{~K}_{*}^{\mathrm{top}}(X \rtimes G ; A)[W]$,

and that our forgetful homomorphism $\mathcal{F}: \mathrm{K}_{*}^{\mathrm{top}}(X \rtimes G ; A) \rightarrow \mathrm{K}_{*}^{\mathrm{top}}(G ; A)$ can be computed as the limit over the maps $\mathcal{F}^{W}$, where $W$ runs through the finite subcomplexes of $\mathcal{E}(G / N)$. Thus the proof of Theorem 0.1 will follow from

Proposition 3.5. For each finite subcomplex $W \subseteq \mathcal{E}(G / N)$, the map

$$
\mathcal{F}^{W}: \mathrm{K}_{*}^{\mathrm{top}}(X \rtimes G ; A)[W] \rightarrow \mathrm{K}_{*}^{\mathrm{top}}(G ; A)[W]
$$

is an isomorphism of abelian groups.

Proof. We proceed by induction on $\operatorname{dim} W$, the maximal dimension of the simplices in $W$. If $W$ is a finite union of simplices with $\operatorname{dim} W=n \in \mathbb{N}$, then $|W|$ is a finite union of simplices with $\operatorname{dim}|W|=n-1$. For fixed compact sets $L \subseteq \mathcal{E}(G)$ and $K \subseteq X$ consider the exact sequences

$d: \quad 0 \rightarrow C_{0}(G \cdot(L \times \stackrel{o}{W} \times K)) \rightarrow C_{0}(G \cdot(L \times W \times K)) \rightarrow C_{0}(G \cdot(L \times|W| \times K)) \rightarrow 0$

and

$$
\delta: \quad 0 \rightarrow C_{0}(G \cdot(L \times \stackrel{\circ}{W})) \rightarrow C_{0}(G \cdot(L \times W)) \rightarrow C_{0}(G \cdot(L \times|W|)) \rightarrow 0 .
$$

The construction of Lemma 3.3 applied to $\mathcal{E}(G) \times X$ instead of $X$ provides a completely positive $C_{0}(\mathcal{E}(G) \times X)$-linear (and hence also a $C_{0}(X)$-linear) $G$-equivariant section which by $[20, \S 7]$ (following the ideas of $[2, \S 7]$ ) provides an element

$$
[d] \in \mathcal{R K K}_{1}^{G}\left(X ; C_{0}(G \cdot(L \times|W| \times K)), C_{0}(G \cdot(L \times \stackrel{\circ}{W} \times K))\right)
$$


such that right multiplication by $[d]$ serves as a boundary map in a long exact sequence for the extension $d$ in $\mathcal{R K K}^{G}(X ; \cdot, A)$-theory. Similarly (but this is more easy since no $C_{0}(X)$-linearity is needed here), the extension $\delta$ determines an element

$$
[\delta] \in \mathrm{KK}_{1}^{G}\left(C_{0}(G \cdot(L \times|W|)), C_{0}(G \cdot(L \times \stackrel{\circ}{W}))\right),
$$

which induces a boundary map in $\mathrm{KK}^{G}(\cdot, A)$-theory. By naturality of the map $f$ : $\mathcal{R K K}_{*}^{G}(X ; \cdot, \cdot) \rightarrow \mathrm{KK}_{*}^{G}(\cdot, \cdot)$ which is obtained by forgetting the $C(X)$-structure, the element

$$
f([d]) \in \mathrm{KK}_{1}^{G}\left(C_{0}(G \cdot(L \times|W| \times K)), C_{0}(G \cdot(L \times \stackrel{\circ}{W} \times K))\right)
$$

induces the boundary map in $\mathrm{KK}^{G}(\cdot, A)$-theory corresponding to $d$ (where we simply forget the $C_{0}(X)$-structures - see [20] and [16]). The projection $\pi_{1}: \mathcal{E}(G) \times$ $\mathcal{E}(G / N) \times X \rightarrow \mathcal{E}(G) \times \mathcal{E}(G / N)$ induces a morphism from the extension $d$ to the extension $\delta$, and hence induces a morphism between the long exact sequences in equivariant KK-theory corresponding to $d$ and $\delta$ (see [16]). Together, we obtain a morphism between the six-term sequence in $\mathcal{R} \mathrm{KK}^{G}(X ; \cdot, A)$-theory corresponding to $d$ and the six-term sequence in $\operatorname{KK}^{G}(\cdot, A)$-theory corresponding to $\delta$. Taking limits over $L$ and $K$, we obtain a commutative diagram

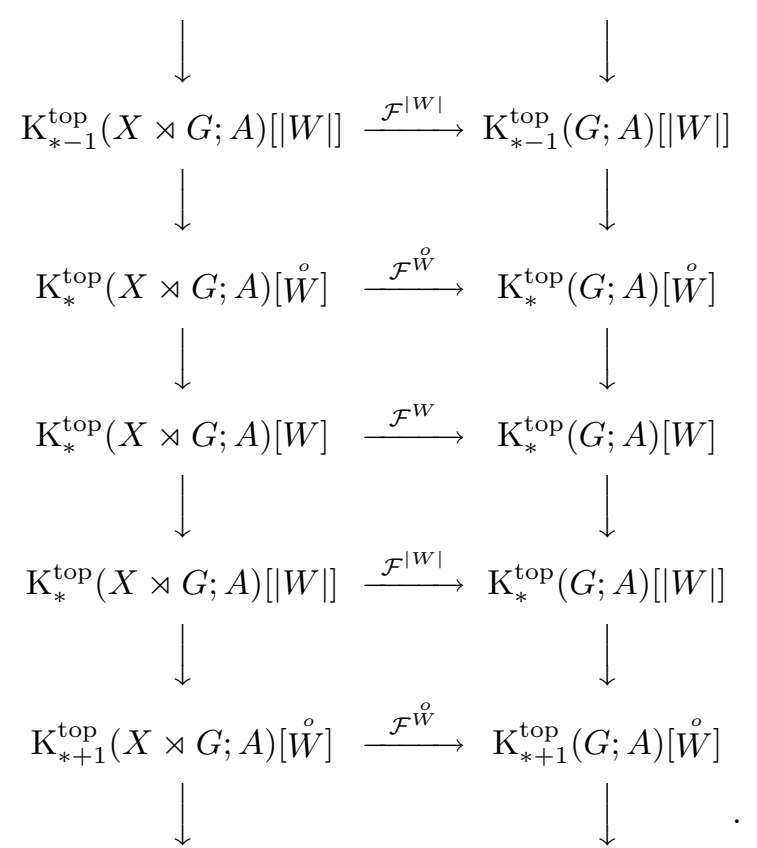

Thus, using the Five Lemma and induction on $\operatorname{dim} W$, the proof reduces to show that

(1) $\mathcal{F}^{W}: \mathrm{K}_{*}^{\mathrm{top}}(X \rtimes G ; A)[W] \rightarrow \mathrm{K}_{*}^{\mathrm{top}}(G ; A)[W]$ is an isomorphism for all zerodimensional finite subcomplexes $W$ of $\mathcal{E}(G / N)$, and 
(2) $\mathcal{F}^{\stackrel{o}{W}}: \mathrm{K}_{*}^{\mathrm{top}}(X \rtimes G ; A)[\stackrel{o}{W}] \rightarrow \mathrm{K}_{*}^{\mathrm{top}}(G ; A)[\stackrel{o}{W}]$ is an isomorphism for all finite subcomplexes $W \subseteq \mathcal{E}(G / N)$.

The proof of (1) is similar to the proof of (2), even a bit simpler. For this reason we only go through the arguments for the latter.

Note that $\stackrel{o}{W}$ is a disjoint union of finitely many open simplices. So we take advantage of the fact that the map respects finite direct sums to reduce to the case where $W$ is a single simplex of dimension $n$. Let $C$ denote the stabilizer of $W$ under the action of $G$. By continuity of the action, $C$ is an open subgroup of $G$. But $G$ acts through $G / N$, so $C$ contains $N$, and since the action of $G / N$ on $\mathcal{E}(G / N)$ is proper, it follows that $C / N$ is compact. Thus, since $N=G_{0}$ is connected, $C$ is an almost connected group and we may apply Lemma 3.1 to $C$ to see that Theorem 0.1 holds for $C$.

Since $C$ is a closed subgroup of $G$, it follows from [4, Remark 6.4] that $\mathcal{E}(G) \times$ $\mathcal{E}(G / N)$ is also a universal example for the proper actions of $C$. Since $C$ is open in $G$ and since $C_{0}(G \cdot(L \times \stackrel{\circ}{W} \times K))=\operatorname{Ind}_{C}^{G} C_{0}(\stackrel{o}{W} \times C \cdot(L \times K))$ (in fact, it is straightforward to check that $G \cdot(L \times \stackrel{\circ}{W} \times K)$ is the $G$-space induced by the $C$ space $\stackrel{\circ}{W} \times C \cdot(L \times K))$, we can apply the compression isomorphism of Proposition 2.2 to obtain the diagram:

$$
\begin{aligned}
& \mathcal{R K K}_{*}^{G}\left(X ; C_{0}(G \cdot(L \times \stackrel{o}{W} \times K)), A\right) \stackrel{\mathcal{F}^{W}}{\longrightarrow} \mathrm{KK}_{*}^{G}\left(C_{0}(G \cdot(L \times \stackrel{o}{W})), A\right) \\
& \mathcal{R} \mathrm{KK}_{*}^{C}\left(X ; C_{0}(\stackrel{o}{W} \times C \cdot(L \times K)), A\right) \stackrel{\mathcal{F}_{C}^{W}}{\longrightarrow} \mathrm{KK}_{*}^{C}\left(C_{0}(\stackrel{o}{W} \times C \cdot L), A\right) \text {, }
\end{aligned}
$$

where, for convenience, we changed the order of the factors in the direct products of the bottom row. By the properties of the $G$-action on $\mathcal{E}(G / N)$ (see the Notations 3.2), $C$ acts trivially on $\stackrel{\circ}{W}$. Hence, since $\stackrel{\circ}{W} \cong \mathbb{R}^{n}$, we can use Bott periodicity to extend the above diagram as follows:

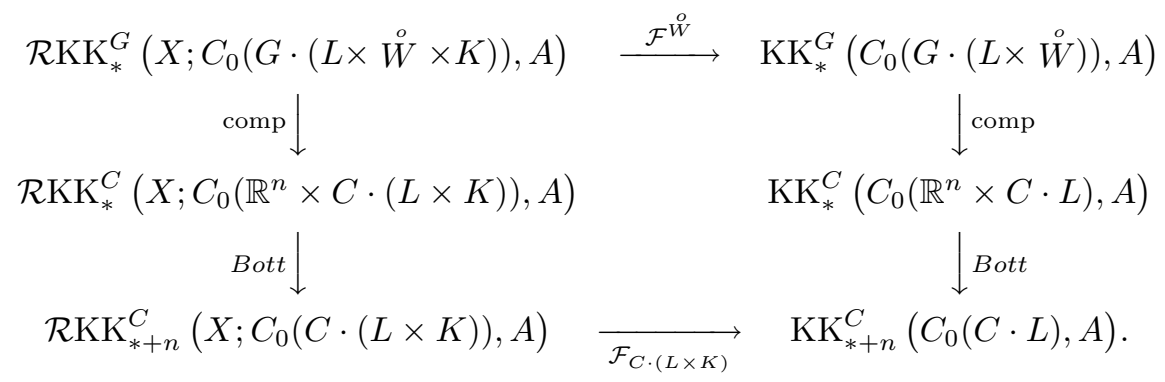

This diagram is commutative and compatible with taking inductive limits over $L$ and $K$. In the limit, the bottom arrow of this diagram becomes an isomorphism by Lemma 3.1. Further, by Bott periodicity and Proposition 2.2 we know that 
the vertical arrows are always isomorphisms. Hence, it follows that the top arrow becomes an isomorphism in the limit. This finishes the proof.

We close this section with some corollaries of the preceding results. The first follows directly from Theorem 0.1 and Lemma 1.2:

Corollary 3.6. Let $X$ be a $G$-space and let $A$ be an $X \rtimes G$-algebra. Then $G$ satisfies $B C$ for $A$ if and only if $X \rtimes G$ satisfies $B C$ for $A$.

The second corollary deals with proper algebras. A $G$-algebra $A$ is called proper if $A$ is an $X \rtimes G$-algebra for some proper $G$-space $X$.

Corollary 3.7. Assume that $A$ is a proper $G$-algebra. Then $G$ satisfies $B C$ for $A$.

The result follows from Corollary 3.6 and

Lemma 3.8. Assume $A$ is an $X \rtimes G$-algebra for some proper $G$-space $X$. Then the assembly map $\mu_{X \rtimes G, A}: \mathrm{K}_{*}^{\mathrm{top}}(X \rtimes G ; A) \rightarrow \mathrm{K}_{*}(A \rtimes G)$ is an isomorphism.

Proof. Since $X$ is a proper $G$-space, it follows easily from the definition of the universal proper spaces that $X$ itself serves as a universal example for proper $X \rtimes G$ spaces. Consider the set of open subsets $V$ of $X$ which have $G$-compact closures $\bar{V}$. Then we can write $A$ as the direct $\operatorname{limit} A=\lim A_{V}$, with $A_{V}:=C_{0}(V) \cdot A$.

For each such $V$ and any $G$-compact subset $Z \subseteq X$ containing $V$, we have a canonical identification

$$
\mathcal{R} \mathrm{KK}^{G}\left(X ; C_{0}(Z), A_{V}\right) \cong \mathcal{R} \mathrm{KK}^{G}\left(Z ; C_{0}(Z), A_{V}\right),
$$

given by the identity map on the cycles $(\mathcal{E}, \Phi, F)$. Since $Z$ is $G$-compact, it follows from $\left[12\right.$, Theorem 5.4] (see also [22, Proposition 1.1]) that $\mathcal{R} \mathrm{KK}^{G}\left(X ; C_{0}(Z), A_{V}\right) \cong$ $\mathrm{K}_{*}\left(A_{V} \rtimes G\right)$ via the assembly map. Taking limits over $Z$ (with $V$ fixed) we see that

$$
\mu_{X \rtimes G, A_{V}}: \mathrm{K}_{*}^{\mathrm{top}}\left(X \rtimes G ; A_{V}\right) \rightarrow \mathrm{K}_{*}\left(A_{V} \rtimes G\right)
$$

is an isomorphism for each $V$. Consider now the commutative diagram

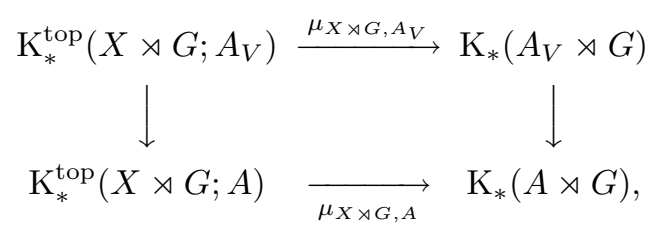

whose upper row is bijective by what we have seen above. Since $A=\lim A_{V}$, it follows from [5, Proposition 7.1] that $\mathrm{K}_{*}^{\mathrm{top}}(X \rtimes G ; A) \cong \mathrm{K}_{*}^{\mathrm{top}}(G ; A) \cong \lim \mathrm{K}_{*}^{\mathrm{top}}\left(G ; A_{V}\right) \cong$ $\lim \mathrm{K}_{*}^{\mathrm{top}}\left(X \rtimes G ; A_{V}\right)$. Thus, passing to the limits on both sides and using the continuity of K-theory, we obtain a diagram where all columns are bijective, too. Hence the bottom line has to be bijective. 
Replacing Lemma 3.8 by a very deep result of $\mathrm{Tu}$ (see [20, Theorem 0.1$]$ ), which says that the Baum-Connes conjecture with coefficients holds for all amenable (Hausdorff) groupoids, Corollary 3.6 can be generalised substantially by

Corollary 3.9. Assume that $X$ is a topologically amenable $G$-space (in the sense of [1]) and that $A$ is any $X \rtimes G$-algebra. Then $G$ satisfies $B C$ for $A$.

\section{Applications to the symmetric imprimitivity theorem}

Let $H$ and $K$ be two locally compact groups and let $X$ be an $H \times K$-space in such a way that the restrictions of the action to the groups $H$ and $K$ on $X$ are free and proper. Let us fix once and for all an $X \rtimes(H \times K)$-algebra $A$. Let $\phi: C_{0}(X) \rightarrow Z M(A)$ denote the corresponding $G$-equivariant homomorphism (see the introduction for the notation). Consider the closed subalgebra of $M(A)$ defined by

$$
\tilde{A}^{K}=\left\{a \in M(A): k \cdot a=a \text { for all } k \in K \text { and } \phi\left(C_{0}(X)\right) a \subseteq A\right\}
$$

and put $A^{K}=\phi\left(C_{0}(K \backslash X)\right) \tilde{A}^{K}$. As a consequence of Cohen's factorization theorem one can show that $A^{K}$ is a closed $H$-invariant subalgebra of $M(A)$, and the obvious homomorphism $\phi^{K}: C_{0}(K \backslash X) \rightarrow Z M\left(A^{K}\right)$ gives $A^{K}$ the structure of an $K \backslash X \rtimes H$-algebra. By symmetry, we can similarly define the $H \backslash X \rtimes K$-algebra $A^{H}$ (cf [10, Definition on p. 164]). In what follows, we say that $A^{K}$ and $A^{H}$ are the generalized fixed-point algebras of $A$ by $K$ and $H$, respectively. Note that if $A=C_{0}(X)$, then we have $A^{K}=C_{0}(K \backslash X)$ and $A^{H}=C_{0}(H \backslash X)$.

It is shown in [10, Theorem 3.15] (but see also [15]) that the crossed products

$$
A^{K} \rtimes_{r} H, \quad A \rtimes_{r}(H \times K), \quad \text { and } \quad A^{H} \rtimes_{r} H
$$

are all Morita equivalent (a similar result holds for the full crossed products). For each $X \rtimes(H \times K)$-algebra $B$, Kasparov constructs in [10, Theorem 3.4] a descent isomorphism

$$
\lambda^{K}: \mathcal{R} \mathrm{KK}_{*}^{H \times K}(X ; B, A) \stackrel{\cong}{\mathcal{R}} \mathrm{KK}_{*}^{H}\left(K \backslash X ; B^{K}, A^{K}\right),
$$

and similarly for $H$ and $K$ reversed. We now want to use this isomorphism to construct natural isomorphisms between the topological K-theories

$$
\mathrm{K}_{*}^{\mathrm{top}}\left(H ; A^{K}\right), \quad \mathrm{K}_{*}^{\mathrm{top}}(H \times K ; A), \quad \text { and } \quad \mathrm{K}_{*}^{\mathrm{top}}\left(K ; A^{H}\right),
$$

respectively. Since the results of the following two lemmas are true without our general second countability assumption, we drop this assumption for those lemmas. 
Lemma 4.1. Let $X \rtimes(H \times K)$ be as above. Suppose that $Z$ is any proper $H$-space. Then $X \times Z$ equipped with the commuting actions of $H$ and $K$ defined by

$$
h \cdot(x, z)=(h \cdot x, h \cdot z) \quad \text { and } \quad k \cdot(x, z)=(k \cdot x, z)
$$

is a proper $H \times K$-space.

Proof. It suffices to show that, whenever $\left(h_{i}, k_{i}\right)_{i \in I}$ is a net in $H \times K$ and $\left(x_{i}, z_{i}\right)_{i \in I}$ is a convergent net in $X \times Z$ such that $\left(h_{i}, k_{i}\right) \cdot\left(x_{i}, z_{i}\right)=\left(h_{i} k_{i} x_{i}, h_{i} z_{i}\right)$ also converges to some $(x, z) \in X \times Z$, then $\left(h_{i}, k_{i}\right)_{i \in I}$ has a convergent subnet. But $\left(h_{i} k_{i} x_{i}, h_{i} z_{i}\right) \rightarrow(x, z)$ implies that $\left(z_{i}\right)_{i \in I}$ is a convergent net in $Z$ with $h_{i} z_{i} \rightarrow z$, and since $H$ acts properly on $Z$, we may pass to a subnet in order to assume that $h_{i} \rightarrow h$ for some $h \in H$. It then follows that $\left(k_{i} x_{i}, z_{i}\right) \rightarrow\left(h^{-1} x, h^{-1} z\right)$, and since $\left(x_{i}\right)_{i \in I}$ is a convergent net in $X$ and $K$ acts properly on $X$ we may pass to another subnet to assume that $\left(k_{i}\right)_{i \in I}$ converges in $K$.

Lemma 4.2. Let $X \rtimes(H \times K)$ be as above. If $Y$ is a proper $X \rtimes(H \times K)$-space, then $K \backslash Y$ is a proper $K \backslash X \rtimes H$-space. The assignment $Y \mapsto K \backslash Y$ determines a natural equivalence between the category of proper $X \rtimes(H \times K)$-spaces and the category of proper $K \backslash X \rtimes H$-spaces with morphisms given by equivariant continuous maps. In particular, if $\mathcal{E}$ is a universal example for the proper $X \rtimes(H \times K)$-actions, then $K \backslash \mathcal{E}$ is a universal example for the proper $K \backslash X \rtimes H$-actions.

Proof. It is straightforward to check the first assertion. To prove the second assertion we define a functor $Z \mapsto X \times_{K \backslash X} Z$ from the category of proper $K \backslash X \rtimes H$ spaces to the category of proper $X \rtimes(H \times K)$-spaces which inverts the functor $Y \mapsto K \backslash Y$ up to isomorphism. Let $q: X \rightarrow K \backslash X$ denote the quotient map and let $p: Z \rightarrow K \backslash X$ denote the structural map for the given $K \backslash X \rtimes H$-action on $Z$. Define

$$
X \times_{K \backslash X} Z=\{(x, z) \in X \times Z: q(x)=p(z)\}
$$

with $H \times K$-action as defined in (4.1) and with structural map $r: X \times_{K \backslash X} Z \rightarrow X$ given by the projection to the first factor. It follows then from Lemma 4.1 that $X \times_{K \backslash X} Z$ is a proper $X \rtimes(H \times K)$-space (since it is a closed subset of $\left.X \times Z\right)$. Moreover, if $\phi: Z \rightarrow Z^{\prime}$ is a continuous $K \backslash X \rtimes H$-equivariant map, then it induces a continuous $X \rtimes(H \times K)$-equivariant map $\phi_{X}: X \times_{K \backslash X} Z \rightarrow X \times_{K \backslash X} Z^{\prime}$ by putting $\phi_{X}(x, z):=(x, \phi(z))$.

It is fairly easy to check that $K \backslash\left(X \times_{K \backslash X} Z\right)$ is $K \backslash X \rtimes H$-equivariantly isomorphic to $Z$ via projection on the second factor. So we only show that $X \times_{K \backslash X}(K \backslash Y)$ is $X \rtimes(H \times K)$-equivariantly isomorphic to $Y$, for any given proper $X \rtimes(H \times K)$ space $Y$. For this let $p: Y \rightarrow X$ denote the corresponding structural map, and let $\dot{p}: K \backslash Y \rightarrow K \backslash X$ denote the map induced by $p$. We are going to define two 
$X \rtimes(H \times K)$-equivariant maps

$$
f: Y \rightarrow X \times_{K \backslash X}(K \backslash Y) \text { and } g: X \times_{K \backslash X}(K \backslash Y) \rightarrow Y
$$

which are inverse to each other. The definition of $f$ is easy: we just put $f(y)=$ $(p(y), K \cdot y)$. To define its inverse $g$ let $(x, K \cdot y) \in X \times_{K \backslash X}(K \backslash Y)$. Then $q(x)=\dot{p}(K \cdot y)=q(p(y))$, where, as above, $q: X \rightarrow K \backslash X$ denotes the quotient map. Since $K$ acts freely and properly on $X$, there exists a unique $k \in K$ such that $x=k \cdot p(y)$ and we define

$$
g(x, K \cdot y):=k \cdot y .
$$

Using the $H \times K$-equivariance of $p$, it is easy to check that $g$ is indeed well-defined, and a short computation shows that $f$ and $g$ are inverse to each other. Thus it only remains to check that $g$ is continuous:

For this let $\left(x_{i}, K \cdot y_{i}\right) \rightarrow(x, K \cdot y)$ in $X \times_{K \backslash X}(K \backslash Y)$. It suffices to show that there exists a subnet $\left(x_{j}, K \cdot y_{j}\right)_{j \in J}$ such that $g\left(\left(x_{j}, K \cdot y_{j}\right)\right) \rightarrow g((x, K \cdot y))$. Since the quotient map $Y \rightarrow K \backslash Y$ is open, we may assume, after replacing $y_{i}$ by an element in the same $K$-orbit and passing to a subnet, that $y_{i} \rightarrow y$. For all $i \in I$ let $k_{i} \in K$ such that $x_{i}=k_{i} \cdot p\left(y_{i}\right)$ and let $k \in K$ such that $x=k \cdot p(y)$. Since $K$ acts properly on $X$, we may assume, after passing to another subnet if necessary, that $\left(k_{i}\right)_{i \in I}$ converges in $K$, and since $K$ also acts freely on $X$ it then follows that $k_{i} \rightarrow k$. Hence

$$
g\left(\left(x_{i}, K \cdot y_{i}\right)\right)=k_{i} \cdot y_{i} \rightarrow k \cdot y=g((x, K \cdot y)) .
$$

The final assertion is now a straightforward consequence of the above.

Remark 4.3. Note that the above result has an easy extension to the case of general actions of $H$. Indeed, exactly the same proof as given above shows that the functor $Y \mapsto K \backslash Y$ determines a natural equivalence between the category of all $X \rtimes(H \times K)$-spaces and the category of all $K \backslash X \rtimes H$-spaces, where the action of $K$ on $X$ is free and proper. Actually, this is an $H$-equivariant version of the pull-back construction for a principal bundle (e.g see [19] I.10).

We should point out, however, that the above lemma and its extension to arbitrary $X \rtimes(H \times K)$-spaces is a very special case of a general construction of Le Gall in [14]. Indeed he shows that whenever we have two (Morita) equivalent groupoids $\mathcal{G}$ and $\mathcal{H}$, then there exists a natural equivalence between the category of $\mathcal{G}$-algebras and the category of $\mathcal{H}$-algebras. In the situation of the equivalent groupoids $X \rtimes(H \times K)$ and $K \backslash X \rtimes H$ one can check that this equivalence is given by the functor $A \mapsto A^{K}$, which, for spaces, restricts to the functor $Y \mapsto K \backslash Y$ as considered above. Since the constructions in [14] are quite technical, it would have taken more time to extract the result of Lemma 4.2 from [14] than presenting the above arguments.

Corollary 4.4. Let $A$ be an $X \rtimes(H \times K)$-algebra. For each $H \times K$-compact subset $Y \subseteq \mathcal{E}(H \times K) \times X$, let 


$$
\lambda_{Y}^{K}: \mathcal{R K K}_{*}^{H \times K}\left(X ; C_{0}(Y), A\right) \rightarrow \mathcal{R K K}_{*}^{H}\left(K \backslash X ; C_{0}(K \backslash Y), A^{K}\right)
$$

denote Kasparov's descent isomorphism of [10, Theorem 3.4]. Then these maps induce a natural (in $A$ ) descent isomorphism

$$
\lambda^{K}: \mathrm{K}_{*}^{\mathrm{top}}(X \rtimes(H \times K) ; A) \rightarrow \mathrm{K}_{*}^{\mathrm{top}}\left(K \backslash X \rtimes H ; A^{K}\right) .
$$

Proof. The result follows directly from Lemma 4.2 and the naturality of Kasparov's descent isomorphism in both variables.

Definition and Remark 4.5. By symmetry, we also get a descent isomorphism

$$
\lambda^{H}: \mathrm{K}_{*}^{\mathrm{top}}(X \rtimes(H \times K) ; A) \rightarrow \mathrm{K}_{*}^{\mathrm{top}}\left(H \backslash X \rtimes K ; A^{H}\right) .
$$

Combining these isomorphisms with the forgetful maps of Theorem 0.1, we obtain a diagram

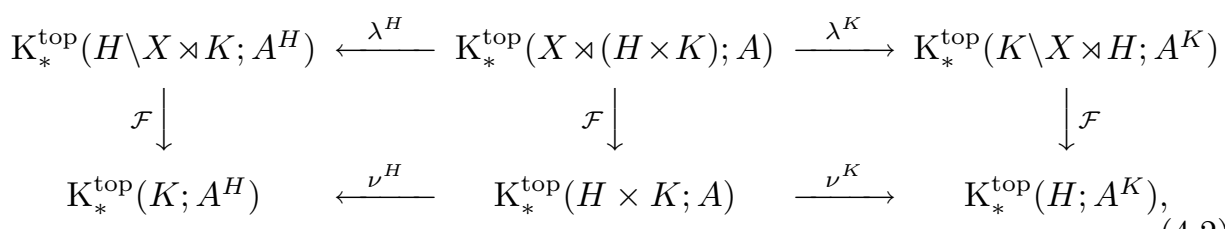

where $\nu^{H}$ and $\nu^{K}$ are chosen such that the left and right squares commute. Since all other maps in the above diagram are isomorphisms, the same is true for the maps $\nu^{H}$ and $\nu^{K}$.

In what follows next, we want to give a direct description of the homomorphisms $\nu^{H}$ and $\nu^{K}$ as defined above. Again, by symmetry, it is enough to do it for $\nu^{K}$. For this let $x \in \mathrm{KK}_{0}^{H}\left(C_{0}(K \backslash Y), C_{0}(Y) \rtimes_{r} K\right)$ be the element given by the fundamental $C_{0}(K \backslash Y)-C_{0}(Y) \rtimes K$-module $\Lambda_{Y, K}^{H \times K, K}$ as defined in [4, Lemma 5.9], and let $y \in \operatorname{KK}_{0}^{H}\left(A \rtimes_{r} K, A^{K}\right)$ denote the invertible element given by the $H$-equivariant Morita equivalence as provided by [10, Theorem 3.13 and 3.15]. Note that if the action of $K$ on $Y$ is also free, then $x$ is also given by a Morita equivalence, but in general we have to deal with arbitrary proper $H \times K$-spaces here. (If $Y$ is actually an $X \rtimes(H \times K)$-space, then the action of $K$ on $Y$ is automatically free!) Now, if $Y$ is any $H \times K$-compact proper $H \times K$-space, we may consider the composition of maps

$\mathrm{KK}_{*}^{H \times K}\left(C_{0}(Y), A\right) \stackrel{J_{K}}{\longrightarrow} \mathrm{KK}_{*}^{H}\left(C_{0}(Y) \rtimes_{r} K, A \rtimes_{r} K\right) \stackrel{x \otimes \cdot \otimes y}{\longrightarrow} \mathrm{KK}_{*}^{H}\left(C_{0}(K \backslash Y), A^{K}\right)$,

which we denote by $\tilde{\nu}^{K}[Y]$. Since $K \backslash Y$ is an $H$-compact proper $H$-space, there exists a continuous $H$-equivariant map $F: K \backslash Y \rightarrow \mathcal{E}(H)$ which induces a homomorphism

$$
F_{*}: \mathrm{KK}_{*}^{H}\left(C_{0}(K \backslash Y), A^{K}\right) \rightarrow \mathrm{K}_{*}^{\mathrm{top}}\left(H ; A^{K}\right) .
$$


It follows then from [5, Lemma 1.5] that the compositions $F_{*} \circ \tilde{\nu}^{K}[Y]$ are natural in $Y$ and hence induce a well defined map

$$
\tilde{\nu}^{K}: \mathrm{K}_{*}^{\mathrm{top}}(H \times K ; A) \rightarrow \mathrm{K}_{*}^{\mathrm{top}}\left(H ; A^{K}\right) .
$$

Remark 4.6. In $[4, \S 5]$ the first two authors constructed a partial assembly map

$$
\mu_{K, A}^{H \times K, K}: \mathrm{K}_{*}^{\mathrm{top}}(H \times K ; A) \rightarrow \mathrm{K}_{*}^{\mathrm{top}}\left(H ; A \rtimes_{r} K\right) .
$$

On the level of a given $H \times K$-compact set $Y$, this map was defined via the composition (denoted $\mu_{K, A}^{H \times K, K}[Y]$ )

$$
\mathrm{KK}_{*}^{H \times K}\left(C_{0}(Y), A\right) \stackrel{J_{K}}{\longrightarrow} \mathrm{KK}_{*}^{H}\left(C_{0}(Y) \rtimes_{r} K, A \rtimes_{r} K\right) \stackrel{x \otimes \cdot}{\longrightarrow} \mathrm{KK}_{*}^{H}\left(C_{0}(K \backslash Y), A \rtimes_{r} K\right)
$$

(see [4, Definition 5.11 and 5.14]). Thus it follows directly from the constructions that

$$
\tilde{\nu}^{K}[Y](z)=\mu_{K, A}^{H \times K, K}[Y](z) \otimes y \quad \text { and } \quad \tilde{\nu}^{K}(u)=\mu_{K, A}^{H \times K, K}(u) \otimes y,
$$

for all $z \in \mathrm{KK}_{*}^{H \times K}\left(C_{0}(Y), A\right)$ and $u \in \mathrm{K}_{*}^{\mathrm{top}}(H \times K ; A)$, respectively.

Proposition 4.7. The maps

$\nu^{K}: \mathrm{K}_{*}^{\mathrm{top}}(H \times K ; A) \rightarrow \mathrm{K}_{*}^{\mathrm{top}}\left(H ; A^{K}\right) \quad$ and $\quad \tilde{\nu}^{K}: \mathrm{K}_{*}^{\mathrm{top}}(H \times K ; A) \rightarrow \mathrm{K}_{*}^{\mathrm{top}}\left(H ; A^{K}\right)$ coincide.

Proof. We have to show that the diagram

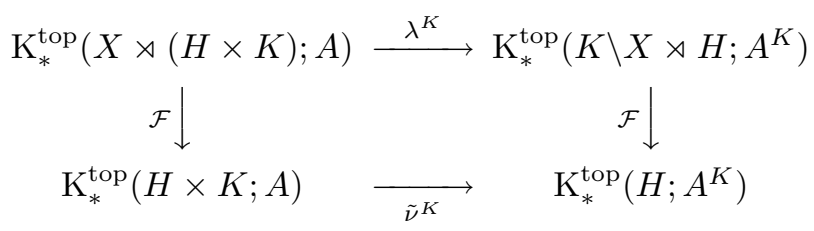

commutes. For this let $Y$ be an $H \times K$-compact proper $X \rtimes(H \times K)$-space. It follows from [10, Theorem 3.14], that the diagram

$$
\begin{array}{rcc}
\mathcal{R K K}_{*}^{H \times K}\left(X ; C_{0}(Y), A\right) & \stackrel{\lambda_{Y}^{K}}{\cong} & \mathcal{R K K}_{*}^{H}\left(K \backslash X ; C_{0}(K \backslash Y), A^{K}\right) \\
J_{K} \circ f & \\
\mathrm{KK}_{*}^{H}\left(C_{0}(Y) \rtimes_{r} K, A \rtimes_{r} K\right) & \underset{x \otimes \cdot \otimes y}{\longrightarrow} & \mathrm{KK}_{*}^{H}\left(C_{0}(K \backslash Y), A^{K}\right)
\end{array}
$$

commutes, where $f: \mathcal{R K K}_{*}^{G}(X ; \cdot, \cdot) \rightarrow \mathrm{KK}_{*}^{G}(\cdot, \cdot)$ is obtained by forgetting the $C_{0}(X)$-structure. Let us map the lower right corner of the diagram into $\mathrm{K}_{*}^{\text {top }}\left(H ; A^{K}\right)$ via any continuous $H$-equivariant map $F: K \backslash Y \rightarrow \mathcal{E}(H)$. Then, if $z \in \mathrm{K}_{*}^{\mathrm{top}}(X \rtimes(H \times K) ; A)$ is represented by an element $z^{\prime} \in \mathcal{R} \mathrm{KK}_{*}^{H \times K}\left(X ; C_{0}(Y), A\right)$, where $Y$ is an $H \times K$-compact invariant subspace of $\mathcal{E}(H \times K) \times X$, the image 
of $z$ in $\mathrm{K}_{*}^{\mathrm{top}}\left(H ; A^{K}\right)$ under the mapping $\mathcal{F} \circ \lambda^{K}$ is given by first applying the upper horizontal arrow to $z^{\prime}$ and then applying the right vertical arrow followed by $F_{*}$. On the other hand, since $\tilde{\nu}^{K}[Y]$ is natural in $Y$, the following diagram is commutative

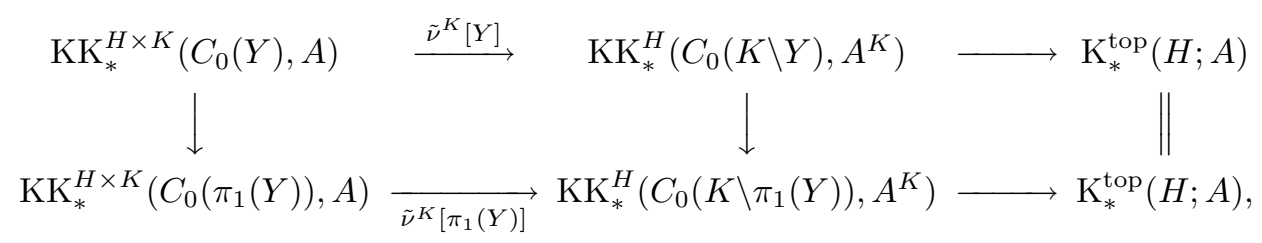

where the first two vertical arrows are induced by $\pi: \mathcal{E}(H \times K) \times X \rightarrow \mathcal{E}(H \times K)$ and the right horizontal arrows are induced by any continuous $H$-equivariant maps $K \backslash Y \rightarrow \mathcal{E}(H)$ and $K \backslash \pi_{1}(Y) \rightarrow \mathcal{E}(H)$. The right square commutes by the universal properties of $\mathcal{E}(H)$ (any two continuous $H$-equivariant maps from $K \backslash Y \rightarrow \mathcal{E}(H)$ are homotopic). Thus, the image of $z$ under the composition $\tilde{\nu}^{K} \circ \mathcal{F}$ is given by first applying the left vertical arrow in diagram (4.5) to $z^{\prime}$ and then the lower horizontal arrow of (4.5) followed by $F_{*}$. This gives the result.

We are now ready to consider the assembly maps. Notice that the following proposition clearly finishes the proof of Theorem 0.5 .

Proposition 4.8. Suppose that $A$ is an $X \rtimes(H \times K)$-algebra. Then the diagram

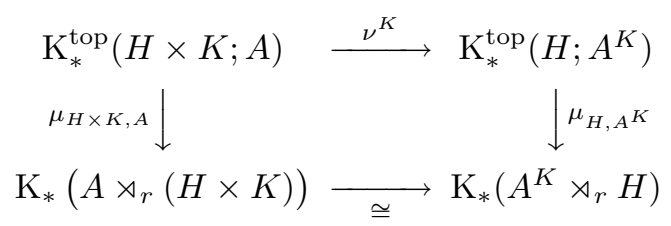

commutes, where the bottom isomorphism is given via the $A \rtimes_{r}(H \times K)-A^{K} \rtimes_{r} H$ Morita equivalence of [10, Theorem 3.15].

Proof. Note that the invertible class in $\mathrm{KK}_{0}\left(A \rtimes_{r}(H \times K), A^{K} \rtimes_{r} H\right)$ corresponding to the equivalence constructed in [10, Theorem 3.15] is just $J_{H}(y)$, where $y \in$ $\mathrm{KK}_{0}^{H}\left(A \rtimes_{r} K, A^{K}\right)$ is the class used in the above explicit construction of $\tilde{\nu}^{K}=\nu^{K}$. Consider the diagram

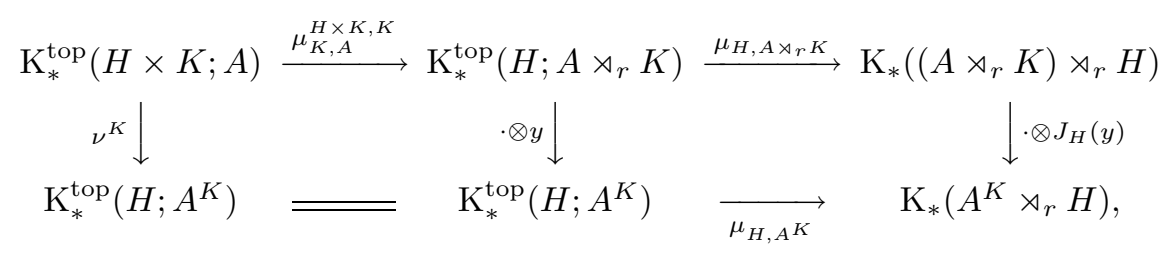

where $\mu_{K, A}^{H \times K, K}: \mathrm{K}_{*}^{\mathrm{top}}(H \times K ; A) \rightarrow \mathrm{K}_{*}^{\mathrm{top}}\left(H ; A \rtimes_{r} K\right)$ denotes the partial assembly map of [4]. It follows from Remark 4.6 that the left square commutes and it follows 
from the compatibility of the assembly map under passing to Morita equivalent coefficient algebras (e.g. see [4, Proposition 5.6]) that the right square commutes. By [4, Proposition 5.15] we know that the composition of the upper horizontal arrows coincides with the assembly map

$$
\mu_{H \times K, A}: \mathrm{K}_{*}^{\mathrm{top}}(H \times K ; A) \rightarrow \mathrm{K}_{*}\left(A \rtimes_{r}(H \times K)\right) \cong \mathrm{K}_{*}\left(\left(A \rtimes_{r} K\right) \rtimes_{r} H\right) .
$$

This finishes the proof.

We are now going to look at the special case of induced algebras. For this suppose that $H$ is a closed subgroup of $G$ and let $B$ be an $H$-algebra. Then, as in $\S 2$, we can define the induced $G$-algebra $\operatorname{Ind}_{H}^{G} B$. In [5] the first two authors constructed a natural induction homomorphism

$$
I_{H}^{G}: \mathrm{K}_{*}^{\mathrm{top}}(H ; B) \rightarrow \mathrm{K}_{*}^{\mathrm{top}}\left(G ; \operatorname{Ind}_{H}^{G} B\right) .
$$

One of the main results of [5] was to show that this homomorphism is always an isomorphism, and that, up to the isomorphism $\mathrm{K}_{*}\left(B \rtimes_{r} H\right) \cong \mathrm{K}_{*}\left(\operatorname{Ind}_{H}^{G} B \rtimes_{r} G\right)$ given by the standard Morita equivalence, it is compatible with the assembly maps. As a consequence, we obtained

Corollary 4.9 (cf [5, Theorem 2.5]). Let $H, G$ and $B$ be as above. Then $H$ satisfies $B C$ for $B$ if and only if $G$ satisfies $B C$ for $\operatorname{Ind}_{H}^{G} B$. In particular, if $G$ satisfies $B C$ for arbitrary coefficients, the same is true for $H$.

We now give an alternative proof of the above result using Theorem 0.5.

Proof of Corollary 4.9. Put $X:=G$ with action of $G$ given by left translation and action of $H$ given by right translation. Then $X \rtimes(H \times G)$ satisfies all requirements of Theorem 0.5. Let $A=C_{0}(X, B)$ viewed as a $X \rtimes(G \times H)$ algebra, with action of $G$ given by $(g \cdot f)(x)=f\left(g^{-1} x\right)$ and $H$-action defined by $(h \cdot f)(x)=$ $h \cdot(f(x h)), f \in C_{0}(X, A)$. It is then fairly straightforward to check that $C_{0}(X, B)^{G}$ is $H$-equivariantly isomorphic to $B$ via evaluation at $e \in G(=X)$, and that $C_{0}(X, B)^{H}$ is $G$-equivariantly isomorphic to $\operatorname{Ind}_{H}^{G} B$. Thus the result follows as a direct consequence of Theorem 0.5 with $G$ playing the role of $K$.

\section{References}

[1] C. Anantharaman-Delaroche and J. Renault, Amenable groupoids, Monographies de L'Enseignement Mathématique 36, Geneva, 2000.

[2] S. Baaj and G. Skandalis, $C^{*}$-algèbres de Hopf et théorie de Kasparov équivariante, Ktheory 2 (1989), 683-721.

[3] P. Baum, A. Connes and N. Higson, Classifying space for proper actions and K-theory of group $C^{*}$-algebras, Contemporary Mathematics 167 (1994), 241-291.

[4] J. Chabert and S. Echterhoff, Twisted equivariant KK-theory and the Baum-Connes conjecture for group extensions, K-Theory 23 (2001), 157-200. 
[5] J. Chabert and S. Echterhoff, Permanence properties of the Baum-Connes conjecture, Doc. Math. 6 (2001), 127-183.

[6] J. Chabert, S. Echterhoff, and Ralf Meyer, Deux remarques sur la conjecture de BaumConnes, C.R.A.S. 332, Série I (2001), 607-610.

[7] N. Higson, Bivariant K-theory and the Novikov conjecture, Geom. Funct. Anal. 10 (2000), $563-581$.

[8] N. Higson and G. Kasparov, E-theory and KK-theory for groups which act properly and isometrically on Hilbert space, Invent. Math. 144 (2001), 23-74.

[9] N. Higson, V. Lafforgue and G. Skandalis, Counterexamples to the Baum-Connes conjecture, GAFA, Geom. funct. Anal. 12 (2002), 330-354.

[10] G. Kasparov, Equivariant KK-theory and the Novikov conjecture, Invent. Math. 91 (1988), $147-201$.

[11] G. Kasparov and G. Skandalis, Groups acting on buildings, operator K-theory, and Novikov's conjecture, K-theory 4 (1991), 303-337.

[12] G. Kasparov and G. Skandalis, Groups acting properly on "bolic" spaces and the Novikov conjecture, Preprint, 1999.

[13] R. Meyer, Equivariant Kasparov theory and generalized homomorphisms, K-theory 21 (2000), 201-228.

[14] P. Y. Le Gall, Théorie de Kasparov équivariante et groupoïdes I, K-theory 16 (1999), 361390.

[15] I. Raeburn, Induced $C^{*}$-algebras and a symmetric imprimitivity theorem, Math. Ann. 280 (1988), 369-387.

[16] G. Skandalis, Exact sequences for the Kasparov groups of graded algebras, Can. J. Math. 37 (1985), 193-216.

[17] G. Skandalis, Progrès récents sur la conjecture de Baum-Connes. Contribution de Vincent Lafforgue, Séminaire Bourbaki, 52ème année, no 869 (1999-2000).

[18] G. Skandalis, J. L. Tu and G. Yu, Coarse Baum-Connes conjecture and groupoids, Topology 41, no 4 (2002), 807-834.

[19] N. Steenrod, The topology of fibre bundles, Princetown University Press, 1951.

[20] J. L. Tu, La conjecture de Novikov pour les feuilletages hyperboliques, K-theory 16 (1999), 129-184.

[21] J. L. Tu, La conjecture de Baum-Connes pour les feuilletages moyennables, K-theory, 17 (1999), 215-264.

[22] J. L. Tu, The Baum-Connes conjecture and discrete group actions on trees, K-theory, 17 (1999), 303-318.

[23] J. L. Tu, The Baum-Connes conjecture for groupoids, In: $C^{*}$-Algebras (J. Cuntz and S. Echterhoff Eds.), Springer, 2000.

[24] A. Valette, Introduction to the Baum-Connes conjecture, from notes taken by Indira Chatterji. With an appendix by Guido Mislin. Lectures in Mathematics ETH Zürich, Birkhäuser Verlag, Basel, 2002.

J. Chabert and H. Oyono-Oyono

Université de Blaise Pascal

Clermont-Ferrand

France

e-mail: chabert@math.univ-bpclermont.fr oyono@math.univ-bpclermont.fr

\author{
S. Echterhoff \\ Westfälische Wilhelms-Universität Münster \\ Mathematisches Institut \\ Einsteinstr. 62 \\ D-48149 Münster \\ Germany \\ e-mail: echters@math.uni-muenster.de
}

(Received: May 10, 2002) 